\title{
Circuit
}

Musiques contemporaines

\section{Entrevue avec André Villeneuve sur l'enjeu de l'identité dans la poïétique musicale}

\section{Danick Trottier}

Volume 15, numéro 2, 2005

Cartes d'identités

URI : https://id.erudit.org/iderudit/902358ar

DOI : https://doi.org/10.7202/902358ar

Aller au sommaire du numéro

Éditeur(s)

Les Presses de l'Université de Montréal

ISSN

1183-1693 (imprimé)

1488-9692 (numérique)

Découvrir la revue

Citer ce document

Trottier, D. (2005). Entrevue avec André Villeneuve sur l'enjeu de l'identité dans la poïétique musicale. Circuit, 15(2), 83-92.

https://doi.org/10.7202/902358ar d'utilisation que vous pouvez consulter en ligne.

https://apropos.erudit.org/fr/usagers/politique-dutilisation/ 


\title{
André Villeneuve
}

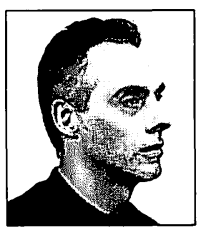

\section{Entrevue avec André Villeneuve sur l'enjeu de l'identité dans la poïétique musicale}

\author{
par Danick Trottier
}

Dans un numéro de Circuit consacré à la question de l'identité, il m'a semblé pertinent de donner la parole et la possibilité au compositeur André Villeneuve de s'exprimer sur sa démarche artistique et sur certains éléments prépondérants dans son langage musical et son rapport à la création musicale contemporaine. Pour ce faire, j'ai souhaité orienter cette réflexion, afin de la mettre en concomitance avec la problématique plus générale de l'identité. Dans cette optique, il me semblait qu'André villeneuve était un exemple privilégié pour plusieurs raisons. D'abord, ayant fréquenté ses classes d'analyse pendant deux ans, j'ai été frappé par l'importance qu'il donnait à la tradition musicale et à l'héritage des maîtres. Les allusions à Claude Ballif, Bruce Mather, Clermont Pépin et Gilles Tremblay étaient des moments forts de son enseignement et faisaient prendre conscience de l'héritage dans lequel l'élève s'inscrivait à son tour. Cela n'est pas sans avoir un impact considérable sur la perception de l'étudiant et sur la manière qu'il conçoit sa formation musicale. Par la suite, après avoir entendu à de nombreuses reprises les œuvres de villeneuve, je savais que ce même passé était pour lui un objet d'intérêt et de questionnement. Par conséquent, il est certainement l'un des compositeurs québécois qui se prête le mieux à cette question de l'attitude à adopter pour le compositeur actuel face au passé musical, source d'inspiration pour plusieurs dans le contexte du postmodernisme artistique. Les références au passé de quelque nature qu'elles soient me semblaient donc une des bases de la relation de Villeneuve à la musique en tant que sujet créateur. Finalement, bien que la génération musicale à laquelle appartient Villeneuve ait fait l'objet de présentations (voir "Québec : Génération fin de siècle", Circuit musiques contemporaines, vol. 10, $n^{0} 1$, 1999), il me semble qu'elle n'a pas encore été questionnée sur ses motivations par rapport au monde musical et sur les réflexions à la base de sa démarche créatrice. Questionner Villeneuve sur les différents aspects de cette dernière semblait l'occasion de combler, du moins en partie, ce déficit. D'un commun accord, nous avons convenu que la meilleure façon d'y parvenir était de réaliser une entrevue écrite, laissant ainsi la possibilité à Villeneuve de réfléchir plus en profondeur aux questions proposées afin d'en arriver à des réponses 
longuement mûries. De mon point de vue, le résultat est probant et je le remercie pour la générosité de ses réponses et pour s'être pleinement prêté au jeu. Les raisons évoquées plus haut allaient donc faire l'objet d'une attention particulière dans la construction du questionnaire en s'articulant autour de l'enjeu de l'identité dans la création musicale actuelle, manière en fin de compte d'avoir un portrait plus juste du compositeur qu'est André Villeneuve.

Danick Trottier : En tant que compositeur, quelles sont les premières idées qui vous viennent à l'esprit lorsqu'il est question de l'identité comme enjeu dans les arts contemporains?

André Villeneuve : Il me vient d'abord une interrogation. Par quel biais, dans le contexte des arts, aborder cette question de l'identité?

S'agit-il ici d'identité de nature sociale, de regroupements, de manières communes, de pratiques artistiques ou de ce qui est propre (l'unicité d'une démarche) à un compositeur? Cela relève du sens que l'on confère au mot "identité".

De prime abord, ce dernier réfère à tout le moins à ce qui est semblable à ceci ou à cela, ou alors il indique le relevé de traits communs. Mais puisqu'il s'agit ici de démarche poiiétique, il me semble que dans le contexte des arts, cette question de l'identité est livrée à l'entendement de chacun. Et, selon chacun, elle se pose ou ne se pose pas.

Pour ma part, si la question se pose, elle doit agir tel un miroir, elle doit renvoyer au questionneur : le compositeur qui s'interroge et interroge sa pratique, ses idées. Et il est alors question d'individualité, de ce qui nous est propre : manière de penser et d'être, manière d'œuvrer. On ne peut questionner l'identité si dans un même élan on ne s'interroge par soi-même (elle ne présenterait alors aucun enjeu) et cela nous ramène alors, invariablement, à la question de l'individualité.

Dans le sens de votre question, je peux concevoir que pour les uns ce questionnement puisse être une affaire de positionnement en regard de courants philosophiques ou esthétiques dans lesquels s'inscrire, se regrouper - associations d'idées ou de pratiques en quelque sorte. Pour d'autres, une manière d'appréhender une actualité changeante, voire même une manière d'être "constant" dans sa façon d'appréhender les choses.

Mais l'art est par nature changeant. Le sont encore plus les pratiques. Je vois mal comment on peut questionner l'identité (sans tomber dans le paradoxe) de ce qui est constamment marqué par le changement (ce qui suppose un constant "ajustement " d'identités, d'opinions ou de pratiques).

Cela revient à dire qu'il ne faut pas confondre la question de l'individualité (de nature intrinsèque) avec celle de l'identification (identité) à ceci ou cela (de nature extrinsèque), toutes deux diamétralement opposées mais dont la seconde (l'identification) nous ramène singulièrement, tôt ou tard, à soi-même (l'individualitê).

D. T. : Pensez-vous que l'identité joue pour beaucoup dans la réflexion artistique et la démarche poïétique que mènent les artistes actuels? 
A. V. : Comment cela en serait-il autrement : en matière d'art tout acheminement de la pensée (œuvrer) et sa matérialité (l'œuvre) porte la marque, aussi discrète soit-elle, de son auteur? Je ne connais pas d'autre credo, qui fut de tout temps et de toute discipline.

Ce questionnement, je l'assimile à l'entreprise d'œuvrer, le "je" qui œuvre, qui est manière d'être et de penser : une manifestation brute de la parole et des idées, qu'elle soit mots, couleurs ou sons. Une individualité qui se manifeste bien au-delà de la question identitaire (s'identifier à).

D'un autre point de vue, dans la société actuelle, au royaume de la sacro-sainte évaluation économique, toute démarche artistique illustre bien la valeur de cette manifestation de l'individualité. Et il suffit de se rappeler qu'en matière d'arts, l'histoire des systèmes et des théories (où l'identification à ceci ou à cela a toujours eu une forte tendance à favoriser les généralisations aux dépends du caractère unique des démarches artistiques) fut toujours bousculée tantôt par ses propres tenants, tantôt par d'autres esprits : ils en furent tour à tour et tout à la fois les concepteurs, les libérateurs ou les fossoyeurs.

D. T. : Je crois ne pas me tromper en affirmant que l'identité est chez vous largement tributaire d'une démarche spirituelle inscrite à tous les niveaux de l'acte poïétique et du rapport en général à la musique. Est-ce exact?

A. V. : Pour qui ne l'est-elle pas si l'on entend par là une "entreprise de l'esprit "? Composer est une quête.

Entre soi et une idée, il y a l'œuvre dans l'intervalle; l'intervalle d'une simple conjonction, ce "et", qui indique l'élan, l'entreprise, le cheminement. Entre soi et une idée, nous œuvrons tous : deux rives à concilier.

Ce et qui est cheminement, depuis l'abstraction originelle de l'idée jusqu'à sa mise en marche temporelle, vibratoire en l'œuvre, se réclame non seulement d'une édification rationnelle par divers moyens techniques propres au langage musical, mais aussi d'une manifestation irrationnelle de la pensée du compositeur où souhaits et intentions d'expressions privilégiées doivent à la fois s'élever et céder aux impératifs des signes, des médiums, de l'interprétation.

Je suis fasciné par cette traversée — dont nous avons la charge - de l'idée vers le signe, par cette poussée qui nous porte vers cet intervalle non mesurable entre intuition et clarté où une idée entrevue deviendra possibilités d'œuvres, par ces divers temps aussi qualifiant notre position : celui qui est antérieur à l'idée, celui-là même de l'idée mise en mouvement, celui de la chute de cette surtension psychique après l'aboutissement, et celui ultérieur des retours à l'entreprise tous qualifiés par la mémoire de ce-qui-a-été-fait.

Ces retours, cette répétition - notre rituel personnel nous replace dans la durée. Et, qu'est-ce qui nous porte à cette répétition, sinon, peut-être, ce voile au-dessus de nos têtes qu'est notre finitude? La première sonorité d'une nouvelle œuvre, son action libératrice, nous allège toujours, momentanément, de ce poids. Ou peut-être est-ce simplement une nostalgie, comme la conscience furtive ou aiguë, a posteriori, d'une heureuse conciliation entre soi et une idée, promesse de lendemains.

D. T. : Vos dernières œuvres, notamment à travers leurs titres latins ou historiques (Ecce ubi sum, pour chœur 
mixte a capella), sont représentatives de cette démarche spirituelle en lien avec une identité en questionnement. Les références à saint Augustin y sont aussi nombreuses. Est-ce pour vous une façon de vous réclamer d'un certain héritage spirituel, pour ne pas dire mystique?

A. V. : J'aime entendre et lire chez certains auteurs la force monolithique de leur engagement. On y perçoit, parfois discrètement ou parfois dans le tumulte, leur désir de se rattacher à une grande Architecture. Je suis sensible à ce désir d'harmonie, encore plus à l'humilité de leur parole puisqu'elle manifeste d'abord ses propres limitations.

Il serait bien prétentieux de dire que je me rattache à un héritage spirituel, encore plus mystique. Dans le cours de "notre petit tracé d'escargot", pour citer Claude Ballif, j'essaie de prendre leçon du parcours de quelques-uns, d'y percevoir leur manière de représentation du monde.

Chez saint Augustin, comme en certaines musiques, tableaux ou poèmes, j'aime retrouver ce désir d'être au sein d'une donnée d'harmonie.

Mais les attributs d'Harmonie relèvent de notre idée d'harmonie.

Pour certains, cela est la Foi : harmonie de conciliation entre soi et ce qui est au-dessus de soi; pour d'autres, l'éthique : harmonie de conciliation entre soi et ses engagements; pour certains, un accord suffira, pour d'autres, le pinceau ou le poème...

En cela, rien ne me touche davantage que l'intime silence de chacun. Pour un tant soit peu qu'on lui prête attention, ce silence nous ramène tous, fondamentalement, à notre condition : que nous sommes mortels mais aussi entourés d'une immensité d'harmonies ou de possibilités d'harmonies portant tous les noms.

D. T. : Dans le même ordre d'idées, dans Les confessions (397-401) de saint Augustin apparaît la prise de conscience du "je” et de l'identité qui le façonne. Comme le dit JeanClaude Guillebaud' en se référant aux réflexions philosophiques sur la genèse du sujet occidental, avec saint Augustin prennent forme trois nouvelles réalités chez le sujet pensant : "la reconnaissance de l'individu comme valeur première", "le siège de la décision éthique est dorénavant l'individu" et l'" affirmation de la liberté intérieure ${ }^{2}$ ). Saint Augustin serait-il pour vous le modèle idéal d'une affirmation de l'identité personnelle dans l'acte créateur?

A. V. : Non pas un modèle mais davantage un témoignage (une prise de la parole au sens éthique) auquel je suis sensible : le questionnement de l'homme sur sa condition, sa quête en écho à ce silence profond, originel, que nous portons tous en nous-mêmes et qui anime notre conscience.

Je pense aussi à Camus, Van Gogh, Tarkowski... la liste est longue et plus près de nous Bruce Mather, Gilles Tremblay, Clermont Pépin... tant de voies différentes.

Et je laisse aux philosophes et aux théologiens le soin de débattre de ce qu'est l'"acte créateur", objet même des mythologies.

La question essentielle étant : Qu'aurons-nous tiré de notre parcours, d'une intention, d'un engagement, d'une idée, d'un simple geste?...

Rilke nous a légué au début d'un simple vers, cette invitation : "Veuille la Transformation de la flamme qui te quitte en gloire de métamorphoses". 
Quel degré alors de cohérence, en regard de soi, en tirons-nous?

Je m'applique à une pratique, à une façon d'œuvrer, celle d'être sonneur, et par là m'instruire du voyage.

D. T. : Venons-en au langage musical. Vous avez publié un article intitulé "Harmonies : déserts fertiles que j'interroge" dans le volume 10, $n^{\circ} 1$ (1999) de Circuit. Ce texte me semble assez révélateur d'un questionnement proprement identitaire vis-à-vis le langage musical et la tradition qui le porte. Est-ce exact?

A. V. : Effectivement, ce texte est révélateur de la nature de mon questionnement. On le retrouve d'ailleurs revu comme premier chapitre de ma thèse de doctorat et par la suite, comme prémisses d'un essai, toujours en cours de rédaction, intitulé Ouvrier d'harmonies.

Le texte de Circuit est ainsi le tout premier où j'exprime mon questionnement sur les notions d'harmonie et d'accord, pour enfin les regrouper sous cette conception sous-jacente à tout mon travail de composition : Harmonie est conciliation dans la distinction.

J'ai aussi compris par la suite, d'une manière plus générale, que ce texte - très heuristique - traduisait mon mode privilégié d'appréhension et d'expression des idées et des choses tantôt par le martèlement du questionnement, tantôt par l'élision qui permet le rapprochement, la mise en relief presque schématique mais toujours révélatrice de la charge historique des notions et des concepts.

Dans ce texte, le langage musical et la tradition sont abordés de biais par le questionnement sur l'harmonie.

Je préfère aussi parler d'héritage au lieu de tradition et par là éveiller en moi un sens plus aigu — il me semble
— de responsabilité en regard de ce-que-nous-avons-reçu. Enfin, toujours assujetti au questionnement sur l'harmonie, j'entends davantage le langage musical comme états et manières d'expressions sonores privilégiées que comme entité en soi. Une façon, en quelque sorte, d'être attentif à ces sonores particuliers que sont les œuvres.

D. T. : Si j'ai bien saisi votre propos contenu dans cet article, l'harmonie semble la voie privilégiée pour questionner l'héritage musical occidental. Mais vous allez encore plus loin : "Harmonie est aussi quête qui se traduit par "éthique" - souhaits de cohérence avec Soi. Harmonie, dans cette acceptation, devient lieux acceptés de nos diversités et de nos contradictions3." N'est-ce pas dans ce cas particulier, c'est-à-dire dans les choix à effectuer par rapport à une tradition donnée, que le compositeur se met au monde en imprégnant le matériau musical de son vécu particulier?

A. V. : On aura vite compris à la lecture de ce texte que je ne m'en suis pas seulement tenu à la notion d'harmonie comme vocabulaire et grammaire d'accords et qu'en abordant cette notion, je ne pouvais éviter de considérer ses représentations en d'autres champs de la pensée.

Harmonie est aussi, selon nos manières de représentations, passage d'un état à un autre; non seulement une quête d'un sonore particulier mais aussi d'un accord (un lien) le plus juste possible entre une idée et le sonore qui lui est propre.

Davantage, cette volonté de conciliation entre l'abstraction d'une idée et sa concrêtude (pour emprunter ce terme à Valéry) réclame en elle-même, purement 
et simplement, un lien de cohérence entre soi et cette même idée. Nous avons le choix de refouler par contentement cette adéquation ou au contraire d'y céder et par là mesurer notre engagement en tant que "sonneur".

D. T. : Dans le cas de Ecce Ubi Sum, vous avez recours à une forme issue du passé, ce qui laisse supposer que vous croyez à une certaine réactualisation des formes anciennes. En quoi l'identité du compositeur actuel, c'est-à-dire d'un être vivant dans un monde bien loin de celui des gens ayant pensé le choral, serait-elle en mesure de refaçonner le choral en lui conférant un nouveau souffle du côté du langage musical?

A. V. : Toute forme en matière d'arts résulte de l'imposition d'une idée. Mais il n'y a pas de perception pure d'une idée : époques, cultures et sociétés, notre propre mémoire nous le démontre bien. Et les formes sont histoires de changement; elles sont sculptées par nos perceptions...

Ce qui me retient, c'est le parcours d'une idée et son tracé se façonnant et prenant forme. À saisir sur la portée de notre imaginaire, est tel ou tel parcours de significations. Une forme devient alors une orientation, un cheminement davantage qu'une nécessité inflexible de parcours.

Dans le cas de ce chœur, Ecce ubi sum, chaque moment est sous l'autorité rythmique des phrases de saint Augustin.

La forme entière de l'œuvre se fait ainsi dans la brièveté d'une phrase additionnée à une autre. Le choral, par son caractère et sa tenue, si près de la parole et du discours, permettait l'installation de chaque phrase d'Augustin comme piliers d'un ensemble.
Et cet ensemble, ici, était le choral; il me fallait tirer parti de cela en réponse à une nécessité, au déploiement d'une idée.

Quant à l'écart chronologique dont vous faites mention - l'usage présent de cette forme et son passé - je répondrai que je ne considère aucune forme héritée comme caduque. Nous sommes les fossoyeurs des formes dans la mesure où on les considère comme des boîtiers ou des vitrines.

Les formes sont en elles-mêmes une mesure du réel qui est mouvement et transformation.

Elles sont acheminement du discours, réclamant tel ou tel contour, telle organisation par besoin de cohérence.

D. T. : Comme le rapportait Henri-Pierre Jeudy, il semble que le créateur contemporain vit un paradoxe au fondement de sa démarche poïétique au regard du passé artistique : "Affirmer sa singularité malgré la répétition des références : telle est la gageure infligée à l'artiste au piège de cette filiation obligée, ordonnée par la notoriété de ces prédécesseurs. Comment être "autre" quand on a le devoir de ressembler à ceux qui nous précèdent ${ }^{4}$ ?" Vivez-vous ce paradoxe dans vos réflexions artistiques et votre démarche poïétique?

A. V. : Ces propos de l'auteur m'étonnent; ils me semblent tirer d'un catalogue de clichés.

Il ne s'agit pas d'être "autre", ni comment être cet "autre", encore moins de manifester le "devoir de ressembler"!

Il s'agit d'être cohérent avec soi et ses idées et d'en suivre le cours. La préoccupation d'être "autre" est 
l'antithèse de toute véritable démarche poïétique, sa négation. II ne faut pas en être dupe. Toute démarche poïétique suppose d'abord un regard sur soi-même.

Et seulement ce regard vif permet de cerner nos positions changeantes qui constituent peu à peu le tracé, le parcours de cette démarche : positions qui sont toujours à délimiter par nos intuitions, par notre imaginaire, par nos références à assimiler...

Tout ce qui nous entoure est histoire de répétitions puisque cela s'inscrit dans le temps. Comment penser se soustraire à une telle richesse!

Je vois là un principe de transformation, de continuité s'il en est : l'invention n'est pas sans référence, sans connaissance de ce qui nous précède.

De croire que cela est de l'ordre d'une "gageure infligée" est absurde : quiconque serait obnubilé par cette dynamique est d'emblée condamner à n'être que par procuration.

II n'en tient qu'à soi de "régler" ses comptes avec ses filiations, de reconnaître avec humilité sa position en regard de ses prédécesseurs et d'en tirer un enseignement : "Avancer vers un but clair avec des yeux clairs" disait le poète Charles van Lerberghe.

Le jeu en vaut la chandelle.

D. T. : Vous insistez beaucoup dans votre enseignement sur l'obligation pour tout musicien de connaître le parcours historique du langage musical et les principes qui l'ont solidifié. De même, vous vous réclamez de l'héritage de vos professeurs : les Claude Ballif, Gilles Tremblay, Bruce Mather. Comment parvenir alors à faire fructifier l'héritage des maîtres tout en arrivant à y mettre du vôtre et à proposer une œuvre originale en soi?
A. V. : Il est un temps où il faut être à la fois réceptif à l'enseignement reçu et du même coup être attentif à établir le territoire de sa réflexion - et de cette manière tirer de l'alchimie des intuitions et voisinages les propres outils de notre langage.

CEuvrer est un tracé solitaire dont notre propre mémoire est initialement enrichie par l'enseignement de nos maîtres.

Il y a le métier et ce que l'on en fait; mais cet héritage dont il est question - outre l'outillage des connaissances transmises - relève aussi de la communication d'un mode de pensée, d'une éthique.

Gilles Tremblay, Bruce Mather, Claude Ballif, fins artisans de la pensée musicale, furent des éclaireurs. L'audition de leurs œuvres est une leçon de musique. Outre la parole nuancée ou le dit du compositeur, c'est précisément leurs musiques qui est héritage. Vous me demandez comment faire fructifier cela? II suffit d'y être pleinement attentif, de porter cela en notre mémoire. Le reste est affaire du voyage solitaire que tous nous devons entreprendre; céder au désir d'édifier et de se retrouver sur le seuil de nos propres idées. On entend toujours mieux sa voix dans le silence de son propre parcours.

D. T. : J'aimerais également vous interrogez sur la problématique de l'identité en création contemporaine par rapport à la société d'appartenance du créateur. II va sans dire que tout créateur, aussi individuelle que soit sa démarche, appartient d'une certaine façon à son époque de par son inscription dans un temps et un lieu donnés : toute époque met en œuvre un Zeitgeist. Dans quelle mesure le compositeur arrive-t-il à participer à ce 
Zeitgeist à la fois en le reflétant et en le dépassant? Dans votre cas précis, croyez-vous qu'une certaine identité propre à votre époque se réverbère dans votre œuvre?

A. V. : Cette appréciation du rapport identité-époque est bien empirique.

Je ne nie pas qu'on puisse en faire l'objet d'un questionnement théorique mais il demeure pour moi essentiellement spéculatif.

Je peux questionner ce rapport mais ne peux l'appréhender par un degré mesurable de participation, car je considère que cette "adhésion " à l'époque ne peut faire l'objet d'une évaluation rationnelle, ce qui serait bien simpliste et réducteur en regard de ce qui est mis en œuvre (au sens propre) par le cheminement de tel compositeur, tel peintre, tel poète... En matière d'arts - entendons de pratiques artistiques - tout est nuance.

Et les œuvres bousculent aisément les constats théoriques, identitaires ou autres vues généralisantes.

Je peux questionner des tendances, des courants, des esthétiques et des "enseignes", me situer par rapport à celles-ci, me renseigner ainsi sur ma position.

Mais l'adhésion ultime et révélatrice se fait à l'instant même où l'on saisit le pinceau ou le crayon.

À cet instant la question de l'identité ne se pose qu'en regard de soi, états changeants de l'être et des idées, dans les limites de son temps bref inscrit dans un temps plus immense.

Toute époque est un état du monde, de choses, de faits, de successions et de transformations qui sont les prolongements d'échos antérieurs en appel de nouveaux lendemains: une trame au sein de laquelle nous faisons face au débit des changements et au cours de laquelle nous ne nous insérons que très brièvement. Je ne suis que dans les limites d'un maintenant toujours changeant et ne peux faire l'expérience que de ce qui est ou de ce qui pourrait être, un à-venir. En cela, comme le dit si bien Cioran, nous sommes "dans la phrase du temps". Et nous sommes tous soulevés par le va-et-vient de ses marées. À moins de vivre en vase clos ou d'être amnésique, comment ne pas être perméable à ce qui nous entoure?

Dans la facture des œuvres se retrouvent toujours ces fines couches où se manifeste tel ou tel signe d'ancrage à une époque.

D. T. : D'une certaine façon, les identités culturelles, sociales, nationales ou locales nous conduisent à des réflexions paradoxales en matière d'arts, pour ne pas dire à des problèmes insolubles. Croyez-vous tout de même, dans le cas par exemple des compositeurs actuels au Québec, que ceux-ci partagent un certain nombre de traits communs repérables dans leurs œuvres?

A. V. : Tant et aussi longtemps que l'on confondra identité et individualité (qui est être et manières d'être) le questionnement identitaire frisera l'absurde.

Tant et aussi longtemps que l'on abordera cette question sous l'angle d'une problématique ou d'un repérage à quantifier, à analyser et sur lequel théoriser, le questionnement identitaire demeurera mort-né car il aura ignoré à tout instant ce "je" qui fait, qui s'achemine, qui œuvre en son propre territoire en coïncidence avec d'autres et dont l'unicité est irréductible à toutes catégories généralisantes. 
Au Québec, comme partout ailleurs, il y a communautés d'esprits et de sensibilités.

Certains échos communs se perçoivent, inévitablement même, au fil des opinions et des écrits, parfois se font entendre lors de l'entreprise de projets communs, et résonnent même parfois au sein des œuvres à tout le moins autour de cafés ou lors de dîners!

Des cheminements s'entrecroisent. Un mot, saisi à la volée, peut donner une œuvre. Nous avons tous en commun un temps si bref, qu'il est heureux qu'il soit nourri par tant d'échanges et balisé par tant de repères. Une idée n'est pas de l'ordre de la propriété.

Et c'est là, en soi, la véritable richesse d'un trait commun, le rebondissement des idées d'un esprit à l'autre et ce qu'elles entraînent dans leur sillage.

D. T. : Pour en arriver au terme de cette entrevue, vous aviez écrit ceci dans l'article de Circuit présenté plus haut : "En matière d'arts, être sur le seuil du questionnement est nécessaires. " Au fond, cette assertion résumerait-elle l'apport identitaire, fruit d'un background particulier, à travers lequel vous cherchez à enchâsser votre démarche poïétique?

A. V. : J'ai bien envie de répondre par une paraphrase : "Je suis donc je questionne".

C'est là ma façon d'être à mon affaire, de suivre le cours d'une idée, de saisir ce qui m'entoure.

Une démarche se fait peu à peu. Le périple est long. Je ne penserais jamais à l'enchâsser. Question d'en conserver l'élan.

\section{NOTES}

1. Guillebaud, J.-C. (1999), La refondation du monde, Paris, Éditions du Seuil.

2. Ibid., p. 292-293.

3. Villeneuve, A. (1999), "Harmonies : déserts fertiles que j'interroge", dans "Génération fin de siècle", Circuit musiques contemporaines, vol. 10, $n^{\circ} 1$, p. 66.

4. Jeudy, H.-P. (1999), Les usages sociaux de l'art, Paris, Ciré, p. 147. 5. Villeneuve, A., op. cit., p. 63. 


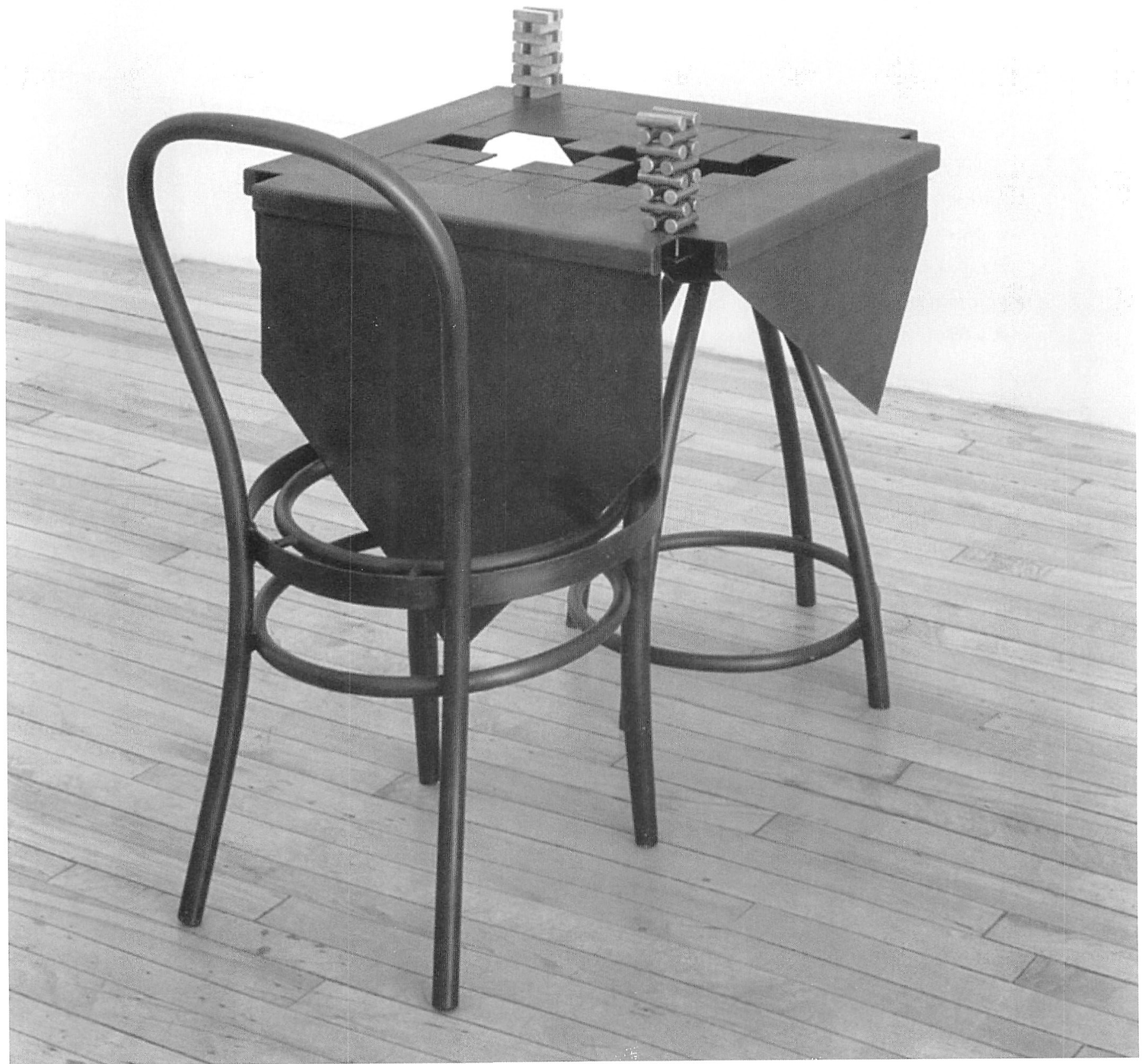

